\title{
Modeling and Simulation of the VideoRay Pro III Underwater Vehicle
}

\author{
Wei Wang and Christopher M. Clark \\ Lab for Autonomous and Intelligent Robotics (LAIR) \\ University of Waterloo, Canada \\ Email: weiwang@engmail.uwaterloo.ca, cclark@mecheng1.uwaterloo.ca
}

\begin{abstract}
Accurate modeling and simulation of underwater vehicles is essential for autonomous control. In this paper, we present a dynamic model of the VideoRay Pro III microROV, in which the hydrodynamic derivatives are determined both theoretically and experimentally, based on the assumption that the motions in different directions are decoupled. The experiments show that this assumption is reasonable within operating conditions of the VideoRay Pro III. A computer simulation with 3D graphics is also developed to help user to visualize the vehicle's motion.
\end{abstract}

\section{INTRODUCTION}

Remotely operated vehicles (ROVs) and autonomous underwater vehicles (AUVs) have been applied in the offshore oil industry, salvage, minehunting, fishery study and other applications where their endurance, economy and safety can replace divers. More recently, there has been a trend to use smaller autonomous vehicles, both tethered and untethered, in lakes and rivers.

Required for autonomous control of such underwater vehicles is a dynamic model. Accurate dynamic models are crucial to the realization of ROV simulators, precision autopilots and for prediction of performance [8] [9].

However, the modeling and control of underwater vehicles is difficult. The governing dynamics of underwater vehicles are fairly well understood, but they are difficult to handle for practical design and control purposes [6] [2]. The problem includes many nonlinearities and modeling uncertainties.

Many hydrodynamic and inertial nonlinearities are present due to coupling between degrees of freedom [3]. For example, currents usually exist in the underwater environment which become coupled with the direction of motion. The presence of these non-linear dynamics requires the use of a numerical technique to determine the vehicle response to thrusters inputs and external disturbances over the wide range of operating conditions.

In general, modeling techniques tend to fall into one of two categories [4]: 1) predictive methods based on either Computational Fluid Dynamics or strip theory, and 2) experimental techniques.

In this paper, a dynamic model of the VideoRay Pro III micro ROV is presented, using both strip theory and experimental techniques. In determining the model parameters, a series of experiments were performed in the Experimental Fluids Lab at the University of Waterloo. These experiments provided data for system identification.

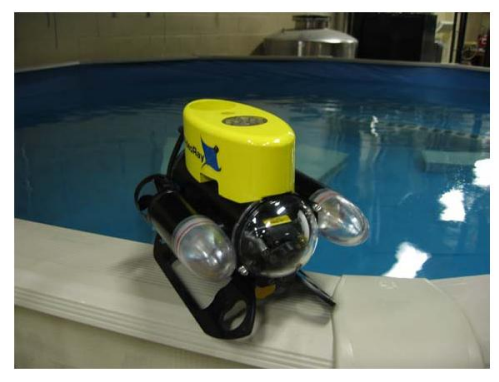

(a) VideoRay Pro III

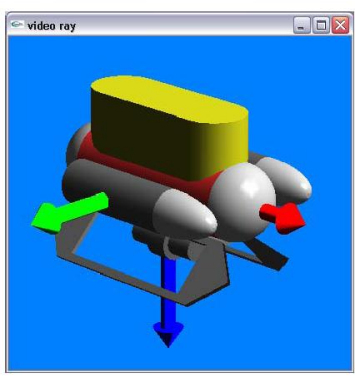

(b) 3D Model
Fig. 1. VideoRay Pro III

The VideoRay Pro III is a small inspection-class personal ROV, with hundreds of units in operation around the world. It is designed for underwater exploration at maximum depth of 500 feet (152 meters) deep. The basic system includes a submersible, an integrated control box, a tether deployment system, and a tool kit. The vehicle has three control thrusters, two of which for horizontal movements, one for vertical movements. It is positive buoyant and hydrostatically stable in the water due to its weight distribution. The vehicle is equipped with a system of sensors including front facing and rear facing cameras, depth gauge and heading meter. Two horizontal thrusters and one vertical thruster are used to control the movement of the VideoRay, (see Fig. 1).

\section{VEHICLE DYNAMICS}

\section{A. 6-DOF Reference Frames}

Underwater vehicle models are conventionally represented by a six degree of freedom, nonlinear set of first order differential equations of motion, which may be integrated numerically to yield vehicle linear and angular velocities, given suitable initial conditions.

The vehicle is considered as a 6 DOF free body in space with mass and inertia, being acted on by numerous forces. Two reference frames are used to describe the vehicles states, one being inertial frame (or earth-fixed frame), one being local body-fixed frame with its origin coincident with the vehicle's center of gravity, and the 3 principle axes in the vehicle's surge, sway and heave directions. (see Fig. 2)

For marine vehicles, the 6 degree of freedom are conventionally defined as surge, sway, heave, roll, pitch and yaw, 
which are defined by the following vectors [3]:

- $\eta=\left[\begin{array}{lllll}x & y & z & \phi & \theta\end{array}\right]^{T}$ : position and orientation (Euler angles) in inertia frame;

- $\nu=\left[\begin{array}{llllll}u & v & w & p & q & r\end{array}\right]^{T}$ : linear and angular velocities in body-fixed frame;

- $\tau=\left[\begin{array}{llllll}X & Y & Z & K & M & N\end{array}\right]^{T}$ : forces and moments acting on the vehicle in body-fixed frame.

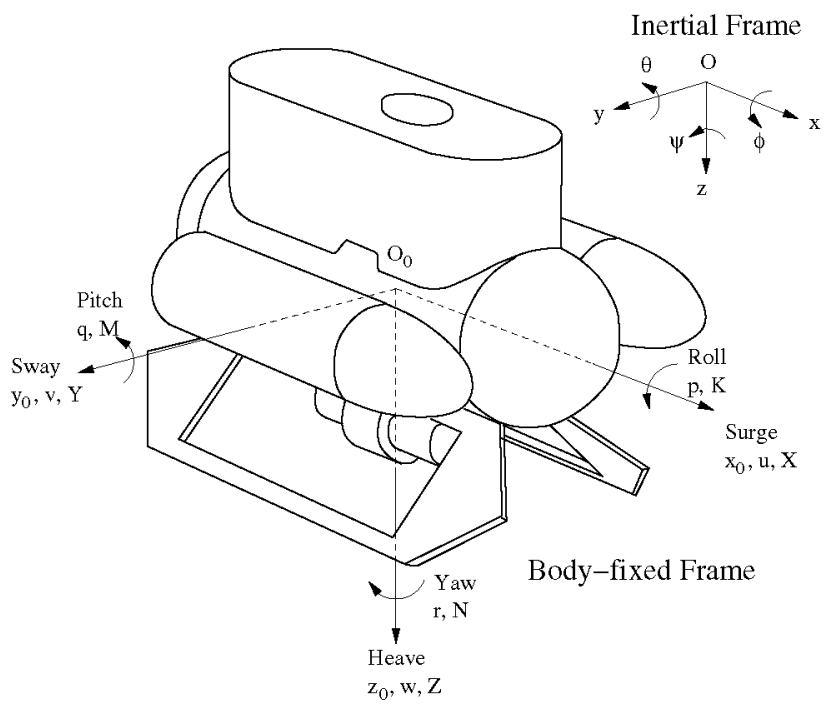

Fig. 2. Body-fixed and inertial reference frames

\section{B. Equations of Motion}

The mathematical model of an underwater vehicle can be expressed, with respect to a local body-fixed reference frame, by a nonlinear equations of motion in matrix form [3]:

$$
\begin{aligned}
\mathbf{M} \dot{\nu}+\mathbf{C}(\nu) \nu+\mathbf{D}(\nu) \nu+\mathbf{g}(\eta) & =\tau \\
\dot{\eta} & =\mathbf{J}(\eta) \nu
\end{aligned}
$$

where:

$\mathbf{M}=\mathbf{M}_{\mathrm{RB}}+\mathbf{M}_{\mathrm{A}}$ is the inertia matrix for rigid body and added mass, respectively;

$\mathbf{C}(\nu)=\mathbf{C}_{\mathrm{RB}}(\nu)+\mathbf{C}_{\mathrm{A}}(\nu)$ is the coriolis and centripetal matrix for rigid body and added mass, respectively;

$\mathbf{D}(\nu)=\mathbf{D}_{\text {quad. }}(\nu)+\mathbf{D}_{\text {lin. }}(\nu)$ is the quadratic and linear drag matrix, respectively;

$\mathbf{g}(\eta)$ is the hydrostatic restoring force matrix;

$\tau$ is the thruster input vector;

$\mathbf{J}(\eta)$ : is the coordinate transform matrix which brings the inertial frame into alignment with the body-fixed frame:

$$
\begin{gathered}
\mathbf{J}(\eta)=\left[\begin{array}{cc}
\mathbf{J}_{1}(\eta) & 0 \\
0 & \mathbf{J}_{2}(\eta)
\end{array}\right] \\
\mathbf{J}_{1}(\eta)=\left[\begin{array}{ccc}
\mathrm{c} \psi \mathrm{c} \theta & -c \psi+c \psi \mathrm{s} \theta \mathrm{s} \phi & \mathrm{s} \psi \mathrm{s} \phi+c \psi \mathrm{s} \theta \mathrm{c} \phi \\
\mathrm{s} \psi \mathrm{c} \theta & c \psi c \phi+\mathrm{s} \psi \mathrm{s} \theta \mathrm{s} \phi & -\mathrm{c} \psi \mathrm{s} \phi+\mathrm{s} \psi \mathrm{s} \theta \mathrm{c} \phi \\
-\mathrm{s} \theta & \mathrm{c} \theta \mathrm{s} \phi & \mathrm{c} \theta \mathrm{c} \phi
\end{array}\right] \\
\mathbf{J}_{2}(\eta)=\left[\begin{array}{ccc}
1 & \mathrm{~s} \phi \mathrm{t} \theta & \mathrm{c} \phi \mathrm{t} \theta \\
0 & \mathrm{c} \phi & -\mathrm{s} \phi \\
0 & \mathrm{~s} \phi / \mathrm{c} \theta & \mathrm{c} \phi / \mathrm{c} \theta
\end{array}\right]
\end{gathered}
$$

Note that $\mathbf{J}_{2}$ above is singular for $\theta= \pm 90^{\circ}$. VideoRay Pro III is unlikely to ever pitch anywhere near $\pm 90^{\circ}$ while underway, and for this reason we choose to define the transformation matrices $\mathbf{J}_{1}$ and $\mathbf{J}_{2}$ in terms of the familiar and widely used Euler angles.

\section{Hydrodynamic Derivatives}

In the vehicle equations of motion (1) and (2), external forces and moments, such as hydrodynamic drag force, actuator thrust, hydrodynamic added mass forces, etc. are described in terms of vehicle's corresponding hydrodynamic coefficients. These coefficients are expressed in the form of hydrodynamic derivative which are in accordance with the SNAME (1950) notation. For example, axial quadratic drag force can be modeled as:

$$
X=-\left(\frac{1}{2} \rho C_{d} A_{f}\right) u|u|=X_{u|u|} u|u|,
$$

which implies that the drag force derivative in surge direction with respect to $u|u|$ is:

$$
X_{u|u|}=\frac{\partial X}{\partial(u|u|)}=-\frac{1}{2} \rho C_{d} A_{f} .
$$

Note that the VideoRay Pro III underwater vehicle is symmetric about the $x-z$ plane, close to symmetric about $y-z$ plane. Therefore, we assume that the motions in surge, sway, pitch and yaw are decoupled [3]. Although it is not symmetric about the $x-y$ plane, the surge and heave motions are considered to be decoupled because the vehicle is basically operated at relative low speed in which the coupling effects can be negligible. For example, with this assumption, the linear drag matrix in Equation (1) is in the form of:

$$
\mathbf{D}_{\text {lin. }}(\nu)=\left[\begin{array}{cccccc}
X_{u} & 0 & 0 & 0 & 0 & 0 \\
0 & Y_{v} & 0 & 0 & 0 & 0 \\
0 & 0 & Z_{w} & 0 & 0 & 0 \\
0 & 0 & 0 & K_{p} & 0 & 0 \\
0 & 0 & 0 & 0 & K_{q} & 0 \\
0 & 0 & 0 & 0 & 0 & N_{r}
\end{array}\right]
$$

A series of experimental tests were performed to verify this assumption and the results indicate that the coupling effects are relatively small and can be neglected. With this assumption and the symmetry property, the resulting added mass matrix and drag matrices will also be diagonal matrices.

\section{Theoretical Parameter Estimation}

Theoretically, the hydrodynamic derivatives can be determined using an approach called strip theory [7]. Fossen [3] provided some two-dimensional added mass coefficients. If the vehicle is divided into a number of strips, the added mass for each 2D strip can be computed and summed over the length of the body to get the 3D hydrodynamic derivative. Besides the added mass, the drag coefficients can also be determined with the application of strip theory. In this way, the hydrodynamic derivatives can be completely determined according to vehicle's geometric properties, even before the vehicle is built. However, the derivatives produced using this approach usually 
can be inaccurate and sometimes unsatisfactory. A validation of these derivatives is always desired.

This approach has been implemented to model the VideoRay's added mass and damping derivatives through the strip theory (see Table I). More importantly, the coefficients in translational directions estimated using strip theory are in good agreement with those later obtained by experiment.

\section{ExPERIMENTAL PARAmeter Identification}

The problem of modeling the VideoRay Pro III is now a matter of estimating and identifying the vehicle's mass, moments of inertia, hydrodynamic derivatives and thruster coefficients in Equation (1). In assuming the motions are decoupled for the VideoRay Pro III, the parameters of interest are the translational drag derivatives in surge, heave, sway directions, and rotational drag derivatives in the yaw direction. These parameters will be determined by experiment.

The inertia matrix in Equation (1) consists of vehicle's mass and the moments of inertia about its three principle axes. In order to estimate the moments of inertia, an oscillation experiment with a small swing angle about vehicle's principle axis was performed. By measuring vehicle's oscillating frequency, the moments of inertia $I_{x x}, I_{y y}$ and $I_{z z}$ can be determined. (see Table I for the results).

Typically, determination of the hydrodynamic derivatives of a vehicle is performed experimentally in towing tank tests or in flumes with controlled flowing water. A series of tests were performed using a flume at the Experimental Fluids Lab in the University of Waterloo. The vehicle is mounted on a horizontal-bending mechanism and submerged in the water. The water flow rate is controlled manually by adjusting the valve positions. The hydrodynamic forces acting on the vehicle is transferred to the horizontal-bending mechanism so that the horizontal force and the bending force can be measured by two load cells respectively. Data is sampled by a data acquisition system and logged by a personal computer. The test setup is depicted in Fig. 3.
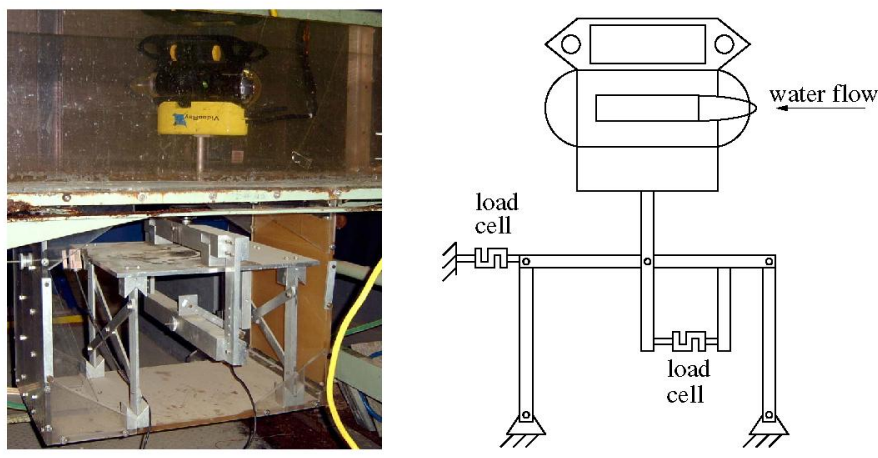

Fig. 3. horizontal-bending mechanism in the flume test

\section{A. Thruster Parameters}

In Equation (1), the thruster input vector $\tau$ consists of the thruster forces and moments acting on the vehicle. This is a function of the thrusters' forces and their current configuration.
An underwater vehicle's thrusters, both for propulsion and directional control, are highly nonlinear actuators. For a fixed pitch propeller, the force (thrust) $T$ depends on the forward speed $u$ of the vehicle, the advance speed $u_{a}$ (ambient water speed), and the propeller rate $n$, (see Fig. 4) as follows [1]:

$$
T=\rho D^{4}\left(\alpha_{1}+\alpha_{2} \frac{u_{a}}{n D}\right) n|n|,
$$

where $\rho$ is the water density, $D$ is the diameter of propeller, $\alpha_{1}$ and $\alpha_{2}$ are constants given by the propeller's property.

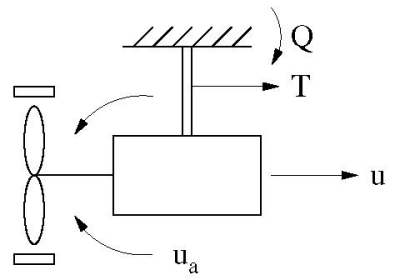

Fig. 4. Schematic drawing of a propeller

A comprehensive study on thrusters and their influence on underwater vehicle maneuverability has been produced [10]. By considering the energy balance of a control volume about a thruster, simplified nonlinear equations for thrust $T$ can be derived as:

$$
\begin{aligned}
\dot{n} & =\beta \tau_{\text {motor }}-\alpha n|n| \\
T & =C_{t} n|n|
\end{aligned}
$$

where $\tau_{\text {motor }}$ is the input torque supplied by the thruster's motor, $\beta, \alpha$ and $C_{t}$ are thruster constants.

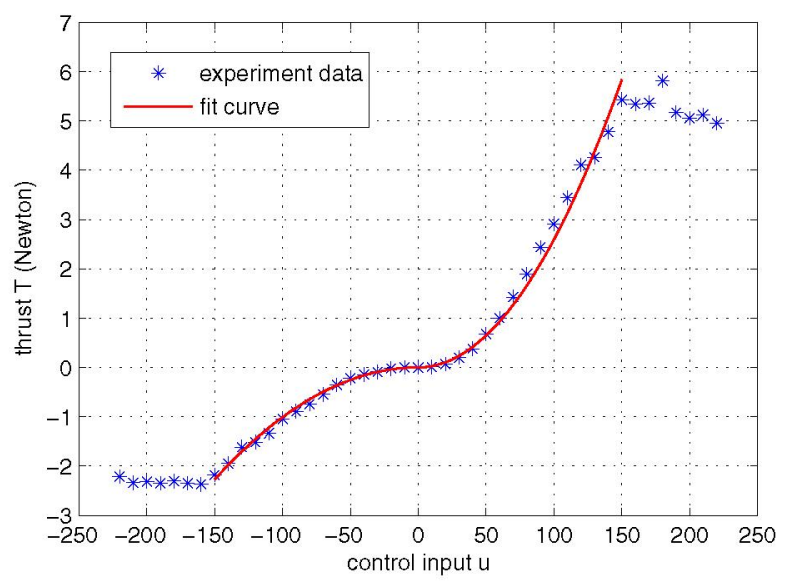

Fig. 5. Output thrust vs. input signal for port/starboard thrusters

The VideoRay Pro III has 3 thrusters: port, starboard and vertical thruster. Each one has its own driver which controls the rotational speed. Since the propeller diameter and mass and their driving motors are small, the dynamics of the thruster control system in Equation (5) is much faster than the dynamics of the vehicle. For this reason, these dynamics are neglected. 
The $C_{T}$ parameter from Equation (6) needs to be identified experimentally. The vehicle was mounted on the horizontalbending mechanism where the thrust of the horizontal thrusters and vertical thruster were measured and recorded at various thruster control signals. Least squares method was applied to compute the coefficients for the port/starboard thrusters and the vertical thruster.

A Mapping of the output thrust versus the thruster input for the two horizontal thrusters is shown in Fig 5. Table I shows the test results.

\section{B. Experimental Set-up for Derivatives in Translational Mo- tions}

Translational hydrodynamic forces in $x, y$ and $z$ directions are modeled as the sum of linear and quadratic terms [3]. For example, the hydrodynamic drag in $x$ direction due to surge motion is expressed as:

$$
\text { Drag Force }=X_{u} u+X_{u|u|} u|u|
$$

where $u$ is the surge velocity, $X_{u}$ is the surge drag force derivative with respect to $u, X_{u|u|}$ is the surge drag force derivative with respect to $u|u|$. When the vehicle moves in low speed, the linear drag term is dominant, while the quadratic drag term is dominant when the vehicle is moving in higher speed. These coefficients account for some entries in the drag matrix $D$ in Equation (1).

In determining the drag coefficients, many flume experiments were performed using the horizontal-bending mechanism to test the drag force under various water flow speeds up to $0.55 \mathrm{~m} / \mathrm{s}$. Fig. 6,7 and 8 show the experiment data and resulting fit curves for the drag forces in surge, sway and heave directions.

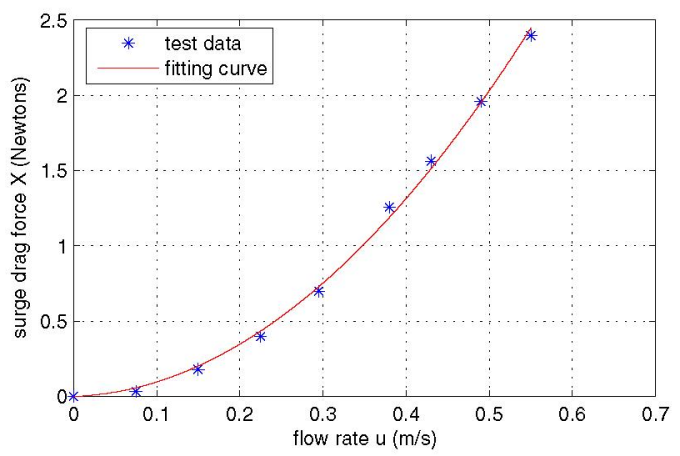

Fig. 6. Drag force in surge direction: experiment data and fit curve

The hydrodynamic forces in heave and sway directions were also tested and recorded while the vehicle is moving in surge direction. Fig. 9 shows the relationship between the change of hydrodynamic force in heave as a function of the surge speed. The results demonstrate that change in heave direction drag force resulting from surge motion are less than one tenth of the drag force in surge direction. Moreover, heave direction drag force resulting from surge motion could be a result of inaccurate positioning of the vehicle during experiments,

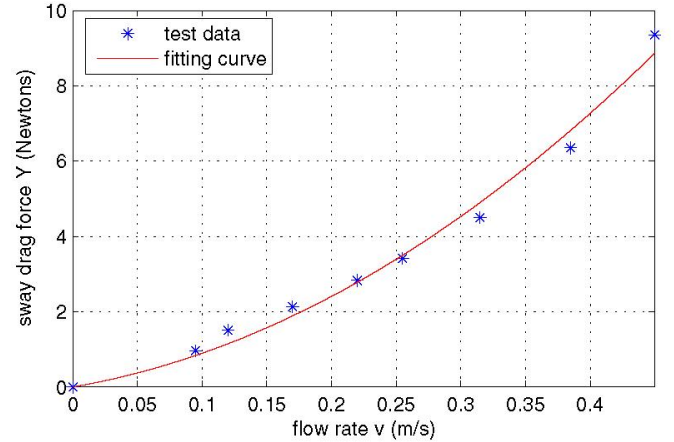

Fig. 7. Drag force in sway direction: experiment data and fit curve

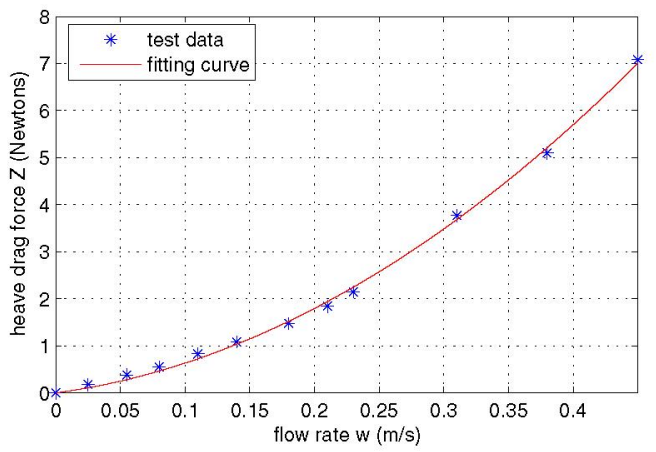

Fig. 8. Drag force in heave direction: experiment data and fit curve

which causes a slight angle of attack with the water flow. Because its magnitude is relatively small, it can be neglected

Fig. 10 shows there is no clear relationship between the sway drag force and the surge speed. This is expected since the vehicle is symmetrical about the $x-z$ plane.

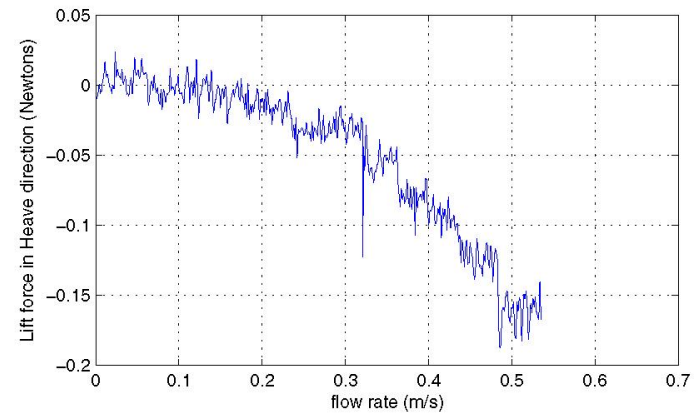

Fig. 9. Heave drag force vs. surge speed

\section{Experimental Set-up and Identification for the Yaw Move- ment}

Accurate hydrodynamic derivatives for the yaw motion is essential for modeling the VideoRay Pro III. Because of the symmetry of $x-z$ and $y-z$ planes, the yaw motion is decoupled from other motions [3]. In this way, the yaw motion can be described by the following model:

$$
\dot{r}=\alpha r+\beta r|r|+\gamma n+\delta
$$




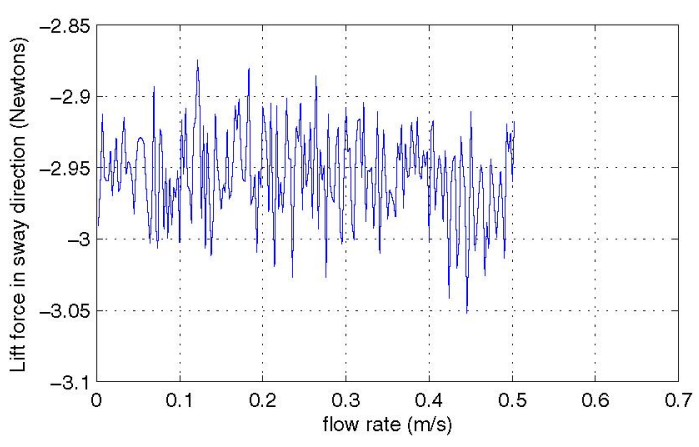

Fig. 10. Sway drag force vs. surge speed

where $r$ is the state variable describing the yaw rate, $n$ is the input variable describing the torque the thrusters exerted on the vehicle, $\alpha$ and $\beta$ are the linear and quadratic drag coefficients, $\gamma$ is the inverse of the vehicle's moment of inertia about $y$ axis, including the rigid body and added mass, and $\delta$ is a bias term. The derivatives $N_{r}, N_{r|r|}$ and $N_{\dot{r}}$, which are part of the entries in the drag matrix in Equation (1), can be derived from $\alpha, \beta, \gamma$ and $\delta$.

The state variable $r$ in Equation (8) is completely controllable by the control variable $\tau$ and completely observable at discrete time instants $\left\{t_{k}\right\}_{k \geq 0}$ through the output variable $y\left(t_{k}\right)$, corrupted by the additive zero-mean noise $e\left(t_{k}\right)$, the system dynamics can be expressed as [5]:

$$
\begin{aligned}
\dot{r} & =\phi(r(t), n(t)) \theta \\
y\left(t_{k}\right) & =r\left(t_{k}\right)+e\left(t_{k}\right)
\end{aligned}
$$

where $\phi(r(t), n(t))=\left[\begin{array}{llll}r & r|r| & n & 1\end{array}\right]$ is a row vector of nonlinear function depending on the state and control input,

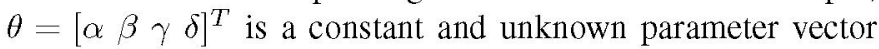
that characterizes the system dynamics.

The identification problem consists of estimating the unknown parameter vector $\theta$ on the basis of a finite number of discrete time measurements of input variable $\left\{n\left(t_{k}\right)\right\}$ and output variable $\left\{y\left(t_{k}\right)\right\}$. The parameter vector $\theta$ can be identified by minimizing the following cost function with the Least Squares method:

$$
J(\theta)=\sum_{k=1}^{N} \epsilon\left(t_{k}\right)^{2}
$$

The cost function is a sum of squares of prediction errors $\epsilon\left(t_{k}\right)$, which are the difference between the observed output variable and the one-step-ahead prediction of the output $\hat{y}\left(t_{k}\right)$ :

$$
\epsilon\left(t_{k}\right)=y\left(t_{k}\right)-\hat{y}\left(t_{k}\right)
$$

If the measurement noise $e\left(t_{k}\right)$ is zero-mean, then the output variable is simplified as:

$$
\hat{y}\left(t_{k}\right)=\hat{r}\left(t_{k}\right)
$$

where $\hat{r}\left(t_{k}\right)$ is the expected state variable at time $t_{k}$.

The one-step-ahead prediction of the output variable $\hat{y}\left(t_{k}\right)$ can be obtained by integrating the state space equation in
Equation (10) between two subsequent time instants $t_{k-1}$ and $t_{k}$ :

$$
r\left(t_{k}\right)-r\left(t_{k-1}\right)=\left[\int_{t_{k-1}}^{t_{k}} \phi(\hat{r}(\tau), n(\tau)) d \tau\right] \theta
$$

From Equation (13), it is implied that $r\left(t_{k-1}\right)=\hat{y}\left(t_{k-1}\right)$. The following estimate for the state variable $r$ at time $t_{k}$ is obtained as:

$$
\hat{r}\left(t_{k}\right)=\hat{y}\left(t_{k-1}\right)+\Phi_{k} \theta
$$

where

$$
\Phi_{k}=\int_{t_{k-1}}^{t_{k}} \phi(\hat{r}(\tau), n(\tau)) d \tau
$$

Hence, the one-step-ahead prediction error of Equation 12 can be evaluated as:

$$
\epsilon\left(t_{k}\right)=y\left(t_{k}\right)-\hat{y}\left(t_{k-1}\right)-\Phi_{k} \theta
$$

Inserting this prediction error into the cost function $J(\theta)$ (Equation 11), we can find out the parameter vector $\theta$ that minimizes the cost function on the basis of $\mathrm{N}$ observations through the Least Squares algorithm:

$$
\theta=\left(\Phi(N)^{T} \Phi(N)\right)^{-1} \Phi(N)^{T} Y(N)
$$

where

$$
\Phi(N)=\left[\begin{array}{c}
\Phi_{1} \\
\Phi_{2} \\
\vdots \\
\Phi_{N}
\end{array}\right], Y(N)=\left[\begin{array}{c}
y\left(t_{1}\right)-\hat{y}\left(t_{0}\right) \\
y\left(t_{2}\right)-\hat{y}\left(t_{1}\right) \\
\vdots \\
y\left(t_{N}\right)-\hat{y}\left(t_{N-1}\right)
\end{array}\right]
$$

The experimental setup for the yaw motion is depicted in Fig. 11. The vehicle is mounted on a pivot which allows the vehicle rotate about its $z$-axis freely. An overhead video camera is placed on top of the vehicle to record its angular movement during the test. The vehicle is driven by the horizontal thrusters with a series of oscillating input signals, which have the same oscillating period and various amplitude from $n=50$ to $n=150$. The vehicle oscillates about its $z$-axis following the input signals. The measured rotational angles of the vehicle are shown in Fig. 12.

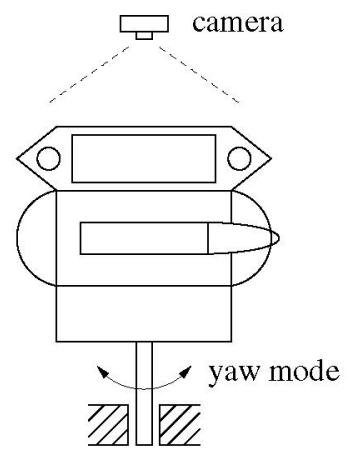

Fig. 11. Experimental set-up for yaw motion

Fig. 13 shows the observed and estimated yaw angle with the thrusters input of $n=150$ and oscillating period $t=1.5$ 


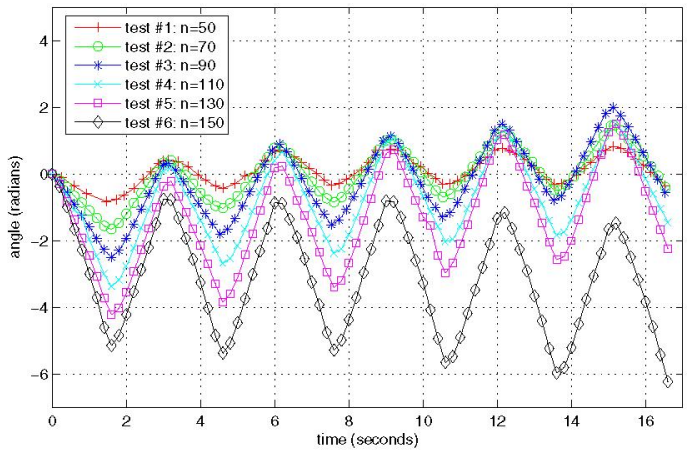

Fig. 12. Test data for yaw motion

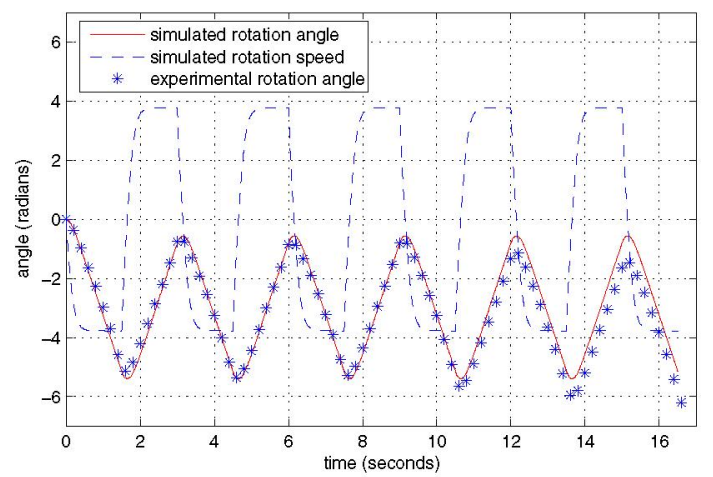

Fig. 13. Identification result for yaw motion with thruster inputs $n= \pm 150$, period $t=1.5$ seconds

seconds. The calculated parameters are: $\alpha=0.6199, \beta=$ $1.1219, \gamma=26.95$ and $\delta=0.0316$.

From the obtained values of $\alpha, \beta$, and $\gamma$, the corresponding hydrodynamic derivatives related to yaw motion $N_{r}, N_{r|r|}$ and $N_{\dot{r}}$ can be derived (see Table I).

\section{MODEL VERIFICATION}

\section{A. Surge Test}

To verify the dynamic model of the VideoRay Pro III, a series of surge tests were performed in a pool. The movements of the vehicle were recorded with a video camera and the distance traveled was analyzed and processed with Matlab. Fig. 14 shows the observed and simulated surge speed with applied thruster input of $n=60$. The predicted surge speed with the dynamic model is $u=0.51 \mathrm{~m} / \mathrm{s}$, which is a bit higher than the actual testing speed of $0.47 \mathrm{~m} / \mathrm{s}$. This could be attributed to the effect of the tether on the vehicle, something not included in our dynamic model.

\section{CONCLUSION}

A hydrodynamic model of the VideoRay Pro III underwater vehicle has been developed theoretically and experimentally, based on the assumption that vehicle motions in different directions are decoupled from one another. A series of experiment tests were performed to verify this assumption. The test

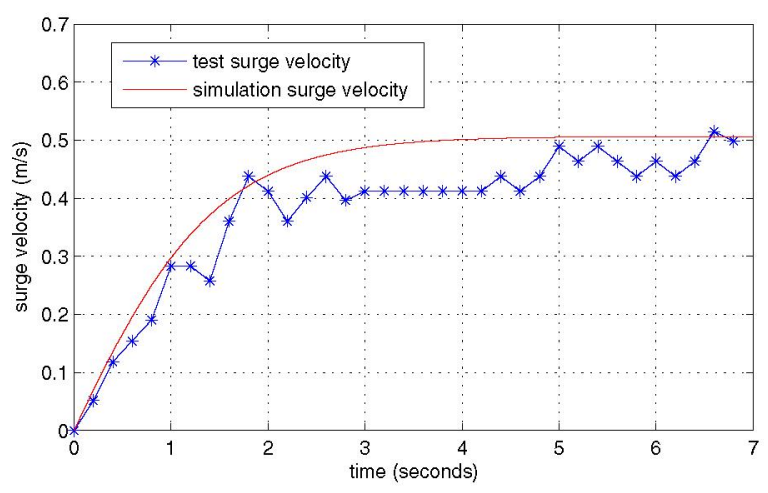

Fig. 14. Surge test experiment data and simulation result

data indicate that this assumption is reasonable within typical operating conditions of the VideoRay Pro III.

In determining the model parameters, several in-flume experiments were performed. For the yaw motion experiment data, a system identification based method was applied to determine the vehicle's hydrodynamic coefficients. Other coefficients were primarily measured, either directly or indirectly with a series of flume tests. The experiments show that the model is in good agreement with the actual test data, despite not including the effect of tether drag. In the future, such effects will be studied and included in the model.

\section{REFERENCES}

[1] M. Blanke. Ship Propulsion Losses Related to Automated Steering and Prime Mover Control. PhD thesis, The Technical University of Denmark, Lyngby, 1981.

[2] M. Caccia, G. Indiveri, and G. Veruggio. Modeling and identification of open-frame variable configuration underwater vehicles. IEEE Joumal of Ocean Engineering, 25.

[3] Thor I. Fossen. Guidance and Control of Ocean Vehicles. John Wiley and Sons Ltd., New York, second edition, 1994.

[4] Kevin R. Goheen. Techniques for urv modeling. 1995.

[5] Lennart Ljung. System Identification: Theory for the User. PTR Prentice Hall, Upper Saddle River, N.J., second edition, 1999.

[6] T. W. McLain and S. M. Rock. Experiments in the hydrodynamic modeling of an underwater manipulator. In Proceedings AUV' 96 , Monterey, CA, 1996.

[7] J. Newman. Marine Hydrodynamics. MIT Press, 8 edition.

[8] Timothy Prestero. Verification of a six-degree of freedom simulation model for the remus autonomous underwater vehicle. Master's thesis, MIT/WHOI Joint Program in Applied Ocean Science and Engineering, 2001.

[9] David A. Smallwood and Louis L. Whitcomb. Toward model based trajectory tracking of underwater robotic vehicles: Theory and simulation. In the 12th International Symposium on Unmanned Untethered Submersible Technology, Durham, New Hampshire, USA, August 2001.

[10] D. R. Yoerger, J. G. Cooke, and J. J. E. Slotine. The influence of thruster dynamic on underwater vehicle behavior and their incorporation into control system design. IEEE Journal of Oceanic Engineering, 15:167168 , July 1990. 
TABLE I

Properties AND COEFFICIENTS For VIDEORAY PRo III

Geometry and mass property

\begin{tabular}{c|c|c|c}
\hline Parameter & Value & Units & Description \\
\hline$L$ & 0.36 & $\mathrm{~m}$ & vehicle length \\
$W$ & 0.35 & $\mathrm{~m}$ & vehicle width \\
$H$ & 0.23 & $\mathrm{~m}$ & vehicle height \\
$I_{x x}$ & 0.02275 & $\mathrm{~kg} \cdot \mathrm{m}^{2}$ & moment of inertia \\
$I_{y y}$ & 0.02391 & $\mathrm{~kg} \cdot \mathrm{m}^{2}$ & moment of inertia \\
$I_{z z}$ & 0.02532 & $\mathrm{~kg} \cdot \mathrm{m}^{2}$ & moment of inertia \\
\hline
\end{tabular}

Thruster coefficients

\begin{tabular}{c|c|c}
\hline \multirow{2}{*}{ thruster } & \multicolumn{2}{|c}{$C_{t}(\mathrm{~N})$} \\
\cline { 2 - 3 } port/starborad & $2.5939 \times 10^{-4}$ & $1.0086 \times 10^{-4}$ \\
\hline vertical & $1.1901 \times 10^{-4}$ & $0.7534 \times 10^{-4}$ \\
\hline
\end{tabular}

Added mass

\begin{tabular}{c|c|c}
\hline & Analytical & Experimental \\
\hline$X_{\dot{u}}$ & 1.9404 & NA \\
$Y_{\dot{v}}$ & 6.0572 & NA \\
$Z_{\dot{w}}$ & 3.9482 & NA \\
$K_{\dot{p}}$ & 0.0326 & NA \\
$M_{\dot{q}}$ & 0.0175 & NA \\
$N_{\dot{r}}$ & 0.0321 & 0.0118 \\
\hline
\end{tabular}

Linear drag coefficients

\begin{tabular}{c|c|c}
\hline & Analytical & Experimental \\
\hline$X_{u}$ & 2.3015 & 0.9460 \\
$Y_{v}$ & 8.0149 & 5.8745 \\
$Z_{w}$ & 5.8162 & 3.7020 \\
$K_{p}$ & 0.0009 & NA \\
$M_{q}$ & 0.0012 & NA \\
$N_{r}$ & 0.0048 & 0.0230 \\
\hline
\end{tabular}

Quadratic drag coefficients

\begin{tabular}{c|c|c}
\hline & Analytical & Experimental \\
\hline$X_{u|u|}$ & 8.2845 & 6.0418 \\
$Y_{v|v|}$ & 23.689 & 30.731 \\
$Z_{w|w|}$ & 20.523 & 26.357 \\
$K_{p|p|}$ & 0.0048 & $\mathrm{NA}$ \\
$M_{q|q|}$ & 0.0069 & $\mathrm{NA}$ \\
$N_{r|r|}$ & 0.0089 & 0.4504 \\
\hline
\end{tabular}

